\title{
El conflicto de Kosovo en la prensa española: del interés al olvido
}

\author{
Javier DíEz ROMERo \\ Universidad Europea de Madrid \\ franciscojavier.diez@uem.es
}

\begin{abstract}
Resumen
La prensa española ha olvidado a Kosovo, a pesar de tratarse de un conflicto al que prestó gran atención durante la guerra de 1999 y los años posteriores. En la mayor parte de los casos, solo se informa de este pequeño territorio balcánico debido a que su proclamación de independencia, llevada a cabo en 2008, aparece cíclicamente en el debate sobre la secesión de Cataluña.
\end{abstract}

Palabras clave: Kosovo; prensa; conflictos olvidados; Cataluña

\section{The conflict of Kosovo in Spanish press: from interest to oblivion}

\begin{abstract}
Currently, the Spanish press has forgotten Kosovo, despite being a conflict to which it paid great attention during the war of 1999 and beyond. Nowadays, in most cases, it only reports about this small Balkan territory due to the fact that its proclamation of independence, carried out in 2008, appears cyclically related to the debate on secession of Catalonia.
\end{abstract}

Key Words: Kosovo; press; forgotten conflicts; Catalonia

Referencia normalizada:

Díez Romero, J. (2013) El conflicto de Kosovo en la prensa española: del interés al olvido. Historia y Comunicación Social. Vol. 18. № Especial Noviembre. Págs. 601-613.

Sumario: 1. Introducción. 2. El conflicto de Kosovo. 3. Kosovo en la prensa española. 3.1 El País. 3.2 El Mundo. 3.3 La Vanguardia. 4. Conclusiones. 5. Bibliografía. 6. Notas

\section{Introducción}

El Instituto de Investigación de Conflictos Internacionales de Heidelberg (Alemania) recoge en su Barómetro de $2012^{1}$ la existencia de cerca de 400 conflictos de diversa intensidad y violencia en todo el mundo. Entre ellos, se encuentra el de Kosovo, la antigua provincia serbia que proclamó su independencia de forma unilateral en 2008. El estudio lo califica de "crisis violenta" y lo sitúa en el tercer nivel, 
en una escala del uno al cinco, considerando esta última cifra la de mayor violencia ${ }^{2}$. Este conflicto enfrenta al gobierno kosovar con la minoría serbia y con la propia Serbia, que reclama la soberanía del territorio.

Para entender la situación actual hay que remontarse a la guerra que tuvo lugar en 1999. Durante 79 días la OTAN bombardeó territorio yugoslavo para presionar a su presidente, Slobodan Milosevic, y detener la limpieza étnica del régimen sobre la mayoría albanesa de la provincia. El conflicto atrajo la atención de los medios de comunicación, que enviaron equipos para informar de unos acontecimientos que volvían a convertir a los Balcanes occidentales en epicentro de la tragedia europea ${ }^{3}$.

El interés de los medios, la clase política y el mundo académico por Kosovo se ha desvanecido, aunque el debate interno sobre la posible secesión de Cataluña y la negativa del Gobierno español a reconocer la independencia de $\mathrm{Kosovo}^{4}$, provoca que la exprovincia serbia siga apareciendo, de forma indirecta, en la política nacional.

\subsection{Objetivos}

La selección de temas por parte de los medios depende, en gran medida, de dinámicas y condicionantes particulares, tales como la cercanía, el interés, la espectacularidad o el simple criterio personal de sus responsables. La audiencia refleja la agenda de temas de los medios. Maxwell McCombs, uno de los padres de la teoría del establecimiento de agenda, lo resume así: "los editores y directores informativos, con su selección día a día y su despliegue de informaciones, dirigen nuestra atención e influyen en nuestra percepción de cuáles son los temas más importantes del día" (2006: 24).

El tsunami de la actualidad ha relegado el conflicto de Kosovo. El presente trabajo analiza las referencias aparecidas entre enero y junio de 2013 en tres diarios de nuestro país para comprobar si ha pasado a ser un conflicto olvidado.

\subsection{Hipótesis}

La hipótesis principal que plantea este estudio es que los medios españoles muestran un reducido interés en Kosovo, conflicto que no consideran relevante. Solo informan o editorializan sobre el pequeño territorio balcánico en momentos puntuales o para dar cuenta del debate soberanista catalán.

\subsection{Metodología}

El estudio se ha llevado a cabo con los periódicos El País, El Mundo y La Vanguardia. Los dos primeros son actualmente los diarios generalistas más leídos de España, mientras que La Vanguardia, además de ser el tercero en número de lectores, tiene su sede en Barcelona y es especialmente sensible a las noticias relacionadas con Cataluña $y$, por tanto, al debate sobre la independencia. 
El objeto del estudio han sido las noticias y artículos de opinión publicados en la versión impresa; posteriormente, se han destacado los más relevantes para la investigación. Asimismo, se han analizado las piezas de la edición en línea que no se publicaron en papel.

\section{EI conflicto de Kosovo ${ }^{6}$}

Desde el final de la Segunda Guerra Mundial, Europa vivió un sueño de unidad del que se despertó violentamente en la década de los noventa en los Balcanes, el lugar al que la Historia se empeña en regresar. La Yugoslavia creada tras la Segunda Guerra Mundial por Josif Broz, Tito ${ }^{7}$, saltó por los aires en 1991. Los nuevos líderes yugoslavos y sus discursos ultranacionalistas llevaron a sus pueblos a la guerra ${ }^{8}$.

La comunidad internacional comenzó a mirar a Kosovo tras la firma de los acuerdos de Dayton, que pusieron fin a la guerra de Bosnia-Herzegovina. Milosevic había anulado el estatuto de autonomía kosovar en 1990. Tras años de protestas, el Ejército de Liberación de Kosovo (ELK) inició en 1998 una guerra de guerrillas y actos terroristas contra la policía serbia. Belgrado respondió con la represión de la población albanokosovar. El 16 de enero de 1999 fueron descubiertos los cadáveres de 45 albaneses en la aldea de Racak. Este hecho aceleró la posterior intervención de la OTAN, ya que la matanza se atribuyó a la policía serbia, aunque "desde el primer momento existieron dudas bastante serias sobre la posibilidad de que el ELK hubiera fabricado el incidente" (Veiga, 2011: 306-307). Tras el ultimátum de la Alianza Atlántica y la fracasada conferencia de Rambouillet (Francia), la OTAN inició los bombardeos el 23 de marzo. La guerra se prolongó hasta la firma del acuerdo de Kumanovo (Macedonia), el 10 de junio, que supuso la retirada serbia.

La Misión de Administración Provisional de las Naciones Unidas en Kosovo (UNMIK, en sus siglas en inglés) ${ }^{9}$ administró la provincia. Aunque seguía perteneciendo de iure a Yugoslavia, los albaneses tomaron el poder de facto. Durante las primeras semanas se desató una vendetta de la comunidad albanokosovar hacia los serbios, mientras la KFOR, la fuerza multinacional de seguridad, miraba hacia otro lado. Decenas de miles de serbios huyeron ante el acoso del ELK ${ }^{10}$, en una nueva campaña de limpieza étnica, esta vez contra los serbios. En 2004 otra ola de violencia acabó con 19 serbios muertos, varios centenares heridos y miles de refugiados ${ }^{11}$.

El enquistamiento de la situación llevó al Enviado Especial de Naciones Unidas a Kosovo, el ex primer ministro de Finlandia Marti Ahtisaari, a elaborar la Propuesta integral de Acuerdo sobre el Estatuto de Kosovo ${ }^{12}$, que "daba luz verde para optar por el camino independentista" (Angoso, 2009: 206).

Las elecciones legislativas y municipales celebradas en Kosovo el 17 de noviembre de 2007 otorgaron la victoria al Partido Democrático de Kosovo, encabezado por el antiguo guerrillero del ELK Hashim Thaçi. Tres meses después, el 17 de febrero 
de 2008, el Parlamento kosovar proclamó la independencia. El nuevo estado fue rápidamente reconocido por Estados Unidos, Alemania, Francia y Reino Unido, mientras que cinco países de la UE, entre ellos España, se manifestaron en contra.

La presión de la Unión Europea sobre Serbia logró que, el 19 de abril de 2013, sus dirigentes llegaran a un acuerdo con los albanokosovares para normalizar sus relaciones.

\section{El conflicto de Kosovo en la prensa española durante el primer semestre de 2013}

Los medios españoles muestran cierto desinterés en el conflicto de Kosovo, según se desprende del análisis de las informaciones y los artículos de opinión aparecidos durante el primer semestre de 2013. Para su estudio, se han establecido cinco categorías: informaciones sobre Kosovo, informaciones sobre política española en las que se hace referencia a la exprovincia serbia, artículos de opinión sobre Kosovo, artículos de opinión sobre política española en los que se alude a la situación de este territorio balcánico y noticias aparecidas solo en la web del periódico (Cuadro 1).

Cuadro 1. Número de referencias sobre Kosovo publicadas entre enero y junio de 2013.

\begin{tabular}{|c|c|c|c|c|c|c|}
\hline \multicolumn{2}{|c|}{ Medio } & Total & $\begin{array}{c}\text { Info } \\
\text { Kosovo }\end{array}$ & $\begin{array}{c}\text { Info } \\
\text { España }\end{array}$ & $\begin{array}{c}\text { Opinión } \\
\text { Kosovo }\end{array}$ & $\begin{array}{c}\text { Opinión } \\
\text { España }\end{array}$ \\
El País & 19 & 8 & 4 & 2 & 5 & 0 \\
\hline El Mundo & 19 & 5 & 5 & 0 & 2 & 7 \\
\hline La Vanguardia & 36 & 5 & 8 & 3 & 2 & 18 \\
\hline
\end{tabular}

Fuente: elaboración propia.

Las referencias publicadas exclusivamente en las ediciones digitales son, en su mayoría, informaciones de agencia y recogen, principalmente, declaraciones de líderes políticos acerca del debate catalán.

\subsection{El País}

El País se caracteriza por mostrar gran atención a la información internacional, pero Kosovo solo aparece en 19 ocasiones, lo que muestra su escaso interés en el conflicto.

\subsubsection{Informaciones sobre Kosovo}

El 7 de febrero de 2013 aparece la primera referencia a Kosovo, con el título "Serbia y Kosovo escenifican el deshielo": 
El deseo de integración en el bloque comunitario no solo promueve reformas en la región, sino que está ablandando incluso la trágica hostilidad entre Serbia y Kosovo [...]. El encuentro culmina un proceso de acercamiento que ha avanzado, bajo los auspicios de Bruselas [...].

Desaparecido Kosovo durante tres meses, hay que esperar al 20 de abril para que $E l$ País publique en portada: "Serbia y Kosovo acuerdan poner fin a su enfrentamiento", gracias a la presión de Bruselas. Dos días después, se actualiza con la información titulada "Bruselas pide ya abrir la candidatura de Serbia a la Unión Europea" y se señalan algunas dificultades:

[...] el proceso se antoja convulso. En primer lugar, aún hay cinco Estados miembros, entre ellos España, que no reconocen la independencia kosovar [...]. Además, los serbios se enfrentan a una fuerte oposición interna.

El 2 de mayo, en el marco de una noticia sobre la ampliación de la Unión Europea, se cita el ejemplo de Kosovo:

Croacia y la zona que representa, los Balcanes, encarnan el aspecto más ilusionante del proyecto europeo: la consolidación de la paz. Porque solo las perspectivas de integración ya propician la concordia, como acaba de ocurrir con el histórico acuerdo de Serbia y Kosovo.

El conflicto vuelve a tener protagonismo el 26 de mayo, con dos crónicas sobre la separación étnica entre serbios y albanokosovares:

La guerra de Kosovo terminó hace 14 años ya, pero en el puente sobre el río Ibar en el centro de Mitrovica todavía se yergue una barricada, cargada de grava, piedras y rencor. [...] La barricada parte en dos la ciudad y todo Kosovo [...].

La última referencia es del 26 de junio y recuerda que Bruselas "ha premiado la reconciliación entre Serbia y Kosovo con un pacto para empezar a negociar en enero de 2014 la entrada de Serbia en la UE”.

\subsubsection{Informaciones sobre política española}

El debate soberanista catalán y las declaraciones de sus protagonistas, en las que a menudo hacen alusión a Kosovo, marcan el ritmo de estas informaciones. La primera aparece el 22 de marzo; en ella, El País recoge unas palabras del portavoz de Convergència i Unió ( $\mathrm{CiU}$ ) en el Congreso, Josep Antoni Duran i Lleida, en las que afirmaba que "Cataluña no quiere ser el Kosovo de turno".

El 19 de abril se informa de que el presidente de la Generalitat de Cataluña, Artur Mas, se manifiesta "a favor del reconocimiento de Kosovo" y, cinco días después, el diario señala que Duran i Lleida no ve "un padrino claro" en el marco internacional que avale el proyecto soberanista. Por último, en la edición del 6 de junio de 2013 se publica otra información, en la que destaca este extracto: 
[Los republicanos] entienden que lo ideal sería que los catalanes pudieran votar sobre la independencia antes del otoño de 2014, cuando se celebrará el referéndum de Escocia. [...] Artur Mas volvió a reivindicar la vía escocesa, [...] y rechazó la vía unilateral que practicó Kosovo.

La noticia recoge las primeras discrepancias públicas entre CiU y Esquerra Republicana de Cataluña acerca del proceso soberanista.

\subsubsection{Artículos de opinión sobre Kosovo}

La publicación en la edición en papel del 23 de abril de la información "Bruselas recomienda iniciar la negociación para la adhesión de Serbia" se ve reforzada con el único editorial que El País dedica a la situación en los Balcanes. "Acuerdo sobre Kosovo" considera el pacto "una esperanza para la estabilidad" y concluye: "los agravios y el odio entre serbios y albanokosovares han crecido sin freno durante años [...]. Pero la barrera psicológica se ha roto".

La segunda referencia se publica el 11 de mayo. La tribuna "Hay otra manera de hacer las cosas", cuya autora es Catherine Ashton, la alta representante de la Unión Europea para los Asuntos Exteriores y la Política de Seguridad, recalca: "los Balcanes occidentales han tenido fama de ser cuna de guerras. Que sean a partir de ahora una cuna de la paz".

\subsubsection{Artículos de opinión sobre politica española}

El director adjunto de El País, Lluís Bassets, publica el 18 de marzo la columna "Federalismo y libertad", en la que señala: "aceptemos que no somos ni queremos ser Kosovo, por más que se empeñen el diario ABC y Soraya Sáenz de Santamaría”.

El 22 de marzo el periódico recurre al historiador Joan B.Culla i Clarà para un artículo de opinión, "De arriba abajo", en el que se analiza la respuesta a la apuesta independentista:

Fue fascinante ver, la semana pasada, cómo uno de esos periódicos se inventaba un supuesto modelo kosovar que inspiraría el proceso político catalán, solo para que, a los tres días, la vicepresidenta Sáenz de Santamaría pudiese glosar la mísera situación de Kosovo y arremeter con ella contra el Gobierno de Cataluña. Eso se llama trabajar en equipo.

El diputado de Amair Jon Iñarritu escribe el 3 de abril una tribuna sobre los procesos soberanistas en el mundo:

[El derecho a decidir] encontraría una de sus máximas justificaciones en el reciente dictamen de la Corte Internacional de Justicia (CIJ) sobre Kosovo, en el cual este tribunal exponía que en el caso de que se agotaran otras vías de entendimiento y se proclamase por los actores políticos legítimos, de manera no violenta, una declaración de independencia, no cabría ningún obstáculo legal internacional. 
La siguiente referencia es una columna de José Ignacio Torreblanca. El autor pide reconocer la independencia de Kosovo y considera que este reconocimiento no se produce por "los efectos debilitantes" del debate catalán. Torreblanca se pregunta: “¿Dónde está el precedente? En la estupidez humana [...]. Respetemos un poco a los kosovares y mantengámoslos al margen de nuestra política interna".

El último artículo, "Espectros kosovares", también es de Culla i Clarà; en él, el autor critica al gobierno español por su posición respecto a Cataluña:

París, Ottawa, Bruselas y Londres saben abordar sus tensiones territoriales internas desde el diálogo [...] y la expresión libre del voto. España, no. Y esa es, en realidad, la clave de su obsesión enfermiza [...]. Kosovo se ha convertido para la España oficial en el espejo de sus inseguridades, de su miedo a la ruptura interna.

Culla compara así la posición española con la de otros países con conflictos territoriales que sí han reconocido a Kosovo como estado soberano.

\subsection{El Mundo}

Al igual que El País, el diario El Mundo publicó 19 referencias sobre Kosovo, con la salvedad de que en ese número se incluyen las siete que solo aparecieron en la versión digital.

\subsubsection{Informaciones sobre Kosovo}

La escasez de informaciones sobre la exprovincia serbia es tal que hasta el 11 de marzo no hay una noticia directa. El texto recoge unas declaraciones del antiguo jefe del Eurogrupo, Jean-Claude Juncker, en las que afirma: "los demonios de una posible guerra en Europa no han desaparecido, «están solo durmiendo, como demostró la guerra en Bosnia y Kosovo»".

El Mundo publica su segunda noticia el 20 de abril: "Serbia y Kosovo tienden ahora un puente histórico. Sellan un acuerdo que reconoce de forma implícita la independencia kosovar". En ella se recuerda que el pacto concede "cierta autonomía para la región al norte de Kosovo de mayoría serbia [y] el reconocimiento implícito de Belgrado de la autoridad de Pristina".

Seis días después, recoge que el presidente serbio, Tomislav Nikolic se puso "De rodillas para pedir perdón por Srebrenica", en alusión a la matanza ocurrida en la ciudad bosnia el 11 de julio de 1995. El Mundo recuerda que Nikolic también impulsó el acuerdo con Kosovo.

Finalmente, el 26 de junio, el diario considera que "España acepta a Kosovo como socio", aunque no lo reconoce como estado. 


\subsubsection{Informaciones sobre España}

Aunque la primera noticia -sobre la reducción de tropas españolas en el exterioraparece el 6 de enero, es el día 24 cuando se publica la primera información en la que se relaciona a Kosovo con la política española. A partir del titular "No es un «proceso descolonizador» ni un «pueblo oprimido»", se recoge un informe de la Asesoría Jurídica Internacional del Ministerio de Asuntos Exteriores respecto a Cataluña. El texto rechaza comparaciones con el caso de Kosovo:

No puede resultar baladí la diferencia palmaria entre un territorio que ha sido objeto de atrocidades contrarias a los más elementales postulados del Derecho Internacional, con un territorio en el que se aplica una Constitución democrática basada en el Estado de Derecho y en el que se reconoce un muy alto grado de descentralización $[\ldots]$.

El 19 de abril el periódico muestra las divergencias existentes en CiU. Al finalizar la pieza, llega la referencia a la exprovincia serbia: "Mas lamentó ayer que España «siga sin reconocer a Kosovo»". En esta misma línea, el día 23, el diario refleja la visita de Artur Mas a Bruselas, donde señaló: "Temo que el Gobierno español sea tan duro con el reconocimiento de Kosovo como con el derecho a decidir en Cataluña".

El 9 de mayo, tras la aprobación por parte de la cámara catalana de una Comisión del Estudio del Derecho a Decidir, El Mundo titula: "El PP abandona el Parlament tras denunciar su insumisión". En ese debate, el líder de Ciutadans, Albert Rivera, acusó al Govern y a Esquerra "de tener la independencia unilateral de Kosovo como referente".

\subsubsection{Artículos de opinión sobre España}

El 20 de febrero, Araceli Mangas, catedrática de Derecho Internacional y Relaciones Internacionales de la Universidad Complutense de Madrid, publica el artículo "Los efectos del derecho a decidir", en el que se opone al planteamiento independentista y remite al debate sobre Escocia:

El Reino Unido reconoce que Escocia tiene derecho a separarse si una mayoría de la población lo decide. Pero no acepta que lo decida uno o varios partidos ni el parlamento escocés. Anoten esta plausible idea: nada al estilo Kosovo, como pretende ERC.

También se alude a Kosovo en la columna de Salvador Sostres "Un viejo negocio", del 23 de abril. "Cataluña, como cualquier país o persona, será lo que quiera ser, y si Serbia y Kosovo han llegado finalmente a un acuerdo, cualquier otro acuerdo es posible", afirma el autor.

\subsection{La Vanguardia}

La principal diferencia de La Vanguardia respecto a sus competidores se muestra en la página web: hasta 18 referencias sobre Kosovo se publicaron solo en la edición 
digital; sin embargo, en la versión en papel, el diario catalán presta una atención similar a la de El País, con otras 18 informaciones y artículos de opinión.

\subsubsection{Informaciones sobre Kosovo}

El 24 de marzo La Vanguardia publica su primera información sobre Kosovo, titulada "El magnetismo de la UE acerca a Serbia y Kosovo" y afirma que los esfuerzos de Ashton "podrían fructificar muy pronto y traducirse en un importante acuerdo para dar cierta autonomía a los serbios residentes en Kosovo y borrar los rastros del Estado serbio". El estado de las conversaciones se actualiza el 4 de abril: "Kosovo rechaza una amplia autonomía para los serbokosovares. La UE presiona a Belgrado para que renuncie a controlar la minoría serbia del norte".

El acuerdo final se recoge el 20 de abril, con el titular "Serbia y Kosovo entierran sus diferencias para acercarse a la UE". La información destaca el desmantelamiento de las "estructuras de Estado" serbias en Kosovo:

Las autoridades de Belgrado han aceptado desmantelar estas instituciones paralelas $[\ldots]$ y aceptar la autoridad de Pristina en el norte del país [...]. Kosovo [...] accedió a reconocer cierta autonomía a estos territorios [...].

El 27 de abril el diario barcelonés publica un breve en el que da cuenta de la petición de la UE a Serbia para que "aplique rápido el pacto con Kosovo". Tras dos meses sin referencias directas, La Vanguardia cuenta el 29 de junio: "Merkel impone más condiciones a la negociación con Serbia" para garantizar que Belgrado aplica el acuerdo con Kosovo.

\subsubsection{Informaciones sobre España}

La Vanguardia presta un gran interés a las informaciones sobre el proceso soberanista catalán. El 12 de abril puede leerse una entrevista con Stéphane Dion, un político liberal canadiense, natural de Quebec (Canadá), donde también se vive una pulsión independentista. Con un título clarificador, "Tengo dos identidades y las dos suman", Dion apuesta por el federalismo:

La tentación de una ruptura unilateral con el Estado es grande. Igual que la asimilación nunca funciona, tampoco la ruptura unilateral. El ejemplo es Kosovo. Pero lo han reconocido más de 90 países. Pero solo porque la separación tras la guerra y la intervención de la OTAN era una realidad.

El 16 de abril el periódico titula: "El conseller de Justícia ve imposible la consulta sin un pacto con Madrid". En su desarrollo, señala: "La declaración unilateral la rechazó también Josep Antoni Duran i Lleida, que recordó que «Catalunya no puede hacer como Kosovo»".

Un día después, se alerta de la "tensión en el Govern por diferencias en la estrategia para hacer la consulta”. Según la noticia, el líder socialista catalán, Pere Navarro, 
tiene la "convicción" de que el presidente de la Generalitat "quiere para Catalunya una solución como la de Kosovo".

A raíz del acuerdo entre Serbia y Kosovo, La Vanguardia concluye el 20 de abril que supone "más presión para España". Sin embargo, tres días después, se recoge la negativa del ministro español de Asuntos Exteriores, José Manuel García-Margallo, al reconocimiento de Kosovo.

El 24 de abril el diario catalán avanza: "España deniega la petición de la UE de reconocer Kosovo. Rajoy defiende el criterio español pensando en Catalunya". Este subtítulo, con carga editorial, relaciona el proceso independentista catalán con la negativa española.

La última referencia aparece el 26 de junio: "España permite a la UE negociar con Kosovo pese a no reconocer al país". En su desarrollo se dice:

El Gobierno español levantó ayer sus reservas al plan de la UE de estrechar lazos con Kosovo [...]. La fórmula jurídica [...] no supone ningún reconocimiento "ni implícito ni explícito" de la independencia del país, aseguró el secretario de Estado de Asuntos Europeos, Íñigo Méndez de Vigo, en Luxemburgo.

La información añade que el propio Méndez de Vigo anunció que se mantendrá la posición de no reconocimiento.

\subsubsection{Artículos de opinión sobre Kosovo}

La primera referencia de opinión sobre Kosovo se publica el 24 de abril. Es un suelto ${ }^{13}$ que refleja que Kosovo "se conformaría con negociar un acuerdo de estabilización" con la UE.

Un mes después, el 30 de abril, en la sección de opinión Los semáforos, se otorga el semáforo verde al presidente de Serbia por sus disculpas al pueblo bosnio y los avances en el diálogo con Kosovo.

Por último, el 30 de junio, La Vanguardia publica un artículo de opinión firmado por Rancois Lenoir, de la agencia Reuters. "Los Balcanes llaman a las puertas de una Europa en crisis existencial" analiza la crisis que vive la UE, la víspera del ingreso de Croacia ${ }^{14}$ y afirma que Kosovo "hace cola para iniciar los trámites" de entrada.

\subsubsection{Artículos de opinión sobre España}

El principio de soberanía aprobado por el Parlament catalán el 23 de febrero es objeto de análisis en un artículo publicado cuatro días después. Su autora, Silvia Hinojosa, recoge los argumentos de Joaquim Ferret, catedrático de Derecho Administrativo de la Universitat Autònoma de Barcelona, sobre el marco legal para celebrar un referéndum: "Por qué Kosovo es independiente? No es comparable al caso catalán, pero lo es por la vía dolorosa de los hechos, y al final por una cuestión política, porque Estados Unidos y Alemania lo reconocieron". 
El 4 de junio, en una carta al director, se critican las palabras de Rajoy acerca de que en Europa "los países pequeños no cuentan" y se señala: "no reconocemos a Kosovo seguramente porque es demasiado pequeño y no cuenta".

\section{Conclusiones}

Durante los primeros meses de 1999 Kosovo se convirtió en centro de la atención informativa de España y del mundo, quizás no tanto por el conflicto entre serbios y albanokosovares, como por la intervención de la OTAN, que bombardeó Yugoslavia en su primera guerra contra un país extranjero desde su nacimiento en 1949.

Kosovo, y también Serbia, continúan viviendo las consecuencias de aquellos días, con un conflicto que se cerró en falso, pero nada queda del interés mostrado por los medios. El presente estudio ha encontrado 74 referencias a la exprovincia serbia en los tres principales periódicos españoles durante los seis primeros meses de 2013. De ellas, solo 27 se publicaron por un interés informativo hacia Kosovo, y la mayoría debido al acuerdo al que llegaron los primeros ministros serbio y kosovar. El resto hacían referencia al debate territorial que se vive en España, con el ejemplo de Kosovo para una posible declaración de independencia de Cataluña.

Kosovo ha pasado a ser un conflicto olvidado para la prensa española, a pesar de situarse geográficamente en el centro de Europa y sufrir un conflicto reciente a gran escala, en el que se vio involucrado nuestro país. El único motivo por el que aparece con cierta frecuencia en los diarios nacionales es por las referencias a su independencia y a la forma en la que se llevó a cabo, comparándola con el proceso iniciado en Cataluña.

\section{Bibliografía}

Libros

ANGOSO GARCÍA, R. (2009): Kosovo. Las semillas del odio. Madrid: Plaza y Valdés.

JUDAH, T. (2008): Kosovo: What everyone needs to know. Reino Unido: Oxford University Press.

MALCOLM, N. (1999): Kosovo: A short story. Estados Unidos: Harper Perennial.

Mc COMBS, M. (2006): Estableciendo la agenda: el impacto de los medios en la opinión pública y en el conocimiento. Barcelona: Paidós, D.L.

TAIBO, C. (1999). Para entender el conflicto de Kosova. Madrid: Los libros de la Catarata. 
VEIGA, F. (2011): La fábrica de las fronteras. Guerras de secesión yugoslavas 19912001. Madrid: Alianza Editorial.

Páginas web

CONFLICT BAROMETER (2012), en Heidelberg Institute for International Conflict Research. Disponible en: http://hiik.de/en/konfliktbarometer/index.html. [1-72013].

REPORT OF THE SPECIAL ENVOY OF THE SECRETARY-GENERAL ON KOSOVO'S FUTURE STATUS, en UNOSEK. Disponible en: http://www. unosek.org/unosek/en/statusproposal.html. [5-7-2013].

\section{Notas}

1 Disponible en http://hiik.de/en/konfliktbarometer/index.html. [1-7-2013].

2 El Instituto de Investigación de Conflictos Internacionales de Heidelberg establece cinco niveles, según su grado de violencia: las guerras y guerras limitadas están consideradas como conflictos de alta intensidad, las crisis violentas son conflictos de intensidad media, mientras que las crisis no violentas y las disputas suponen conflictos de baja intensidad.

3 La desmembración de Yugoslavia en 1991 provocó varias guerras: Eslovenia (1991), Croacia (19911992), Bosnia-Herzegovina (1992-1995), Kosovo (1998-1999) y Macedonia (2001).

4 Un total de 104 países habían reconocido a Kosovo como estado independiente en octubre de 2013, fecha de finalización de este trabajo.

5 Según la segunda oleada del Estudio General de Medios de junio de 2013, El País es el diario generalista más leído, con 1.851.000 lectores, seguido de El Mundo, con 1.150.000, y La Vanguardia, con 748.000 lectores.

6 Desde el estallido de las guerras yugoslavas en 1991, se han publicado decenas de obras sobre el conflicto. Respecto a Kosovo, conviene señalar las obras de Francisco Veiga y Carlos Taibo. Fuera de nuestro país, destacan las monografías de Noel Malcolm y Tim Judah.

7 Josif Broz, Tito, lideró el movimiento partisano comunista que combatió a los nazis en Yugoslavia durante la Segunda Guerra Mundial. Creó un estado federal, con seis repúblicas autónomas, regidas por un sistema de partido único, aunque con ciertas libertades. Durante su mandato se controlaron y reprimieron los movimientos separatistas. Murió en 1980.

8 El conflicto de Eslovenia fue una miniguerra de baja intensidad, que enfrentó a las fuerzas de defensa eslovenas con el Ejército Federal Yugoslavo entre el 26 de junio y el 6 de julio de 1991, causando 80 muertos. La guerra a gran escala en Croacia estalló en agosto de 1991 y se extendió hasta el alto el fuego de enero de 1992, aunque durante los tres años siguientes se produjeron combates aislados. En Bosnia-Herzegovina tuvo lugar el más sangriento de los conflictos yugoslavos: iniciada en abril de 1992, la guerra terminó en octubre de 1995, tras los ataques de la aviación de la OTAN contra posiciones de los serbobosnios. Algunos estudios estiman que estas guerras causaron 200.000 muertos.

9 La UNMIK quedó establecida en junio de 1999, mediante la resolución 1.244 del Consejo de Seguridad, que creaba una administración provisional en Kosovo bajo supervisión internacional. 
10En 1991 vivían en Kosovo unos 200.000 serbios, el 10\% de la población total. Según los datos del censo de 2008, apenas quedan la mitad; ese descenso hay que buscarlo principalmente en las primeras semanas tras la entrada de las fuerzas de la OTAN.

11 No existe consenso sobre el número de serbios que huyeron de Kosovo. Veiga apunta la cifra de 176.000 exiliados hasta julio de 1999 y añade que en los disturbios de 2004 varios miles de serbios "huyeron de sus casas y desaparecieron por completo de ciudades como la capital" (2011: 329). Angoso señala que más de 200.000 serbios y miembros de otras minorías se han ido de Kosovo (2007: 158).

12 Texto en: http://www.unosek.org/unosek/en/statusproposal.html. [5-7-2013].

13 El suelto es una variante menor del editorial. Suele ser breve y refleja la opinión del periódico respecto a temas menores, sobre los que realiza una llamada de atención al lector.

14Croacia ingresó en la Unión Europea el 1 de julio de 2013, convirtiéndose en el socio número 28 del club europeo.

\section{El autor}

Javier Díez Romero es periodista y profesor de la Universidad Europea de Madrid. Compagina su trabajo en el equipo de edición de La Sexta Noticias con la docencia universitaria y la investigación. Sus trabajos se centran en la cobertura de conflictos armados por parte de los medios de comunicación y en la información en televisión. 